\title{
Article \\ Research on the Processing Method of Acoustic Focusing Cavities Based on the Temperature Gradient
}

\author{
Liqun Wu, Yafei Fan, Hongcheng Wang, Linan Zhang, Yizheng Sheng, Yajing Wang and Yaxing Wang *
}

check for updates

Citation: Wu, L.; Fan, Y.; Wang, H.; Zhang, L.; Sheng, Y.; Wang, Y.; Wang, Y. Research on the Processing Method of Acoustic Focusing Cavities Based on the Temperature Gradient. Appl. Sci. 2021, 11, 5737. https://doi.org/ 10.3390/app11125737

Academic Editor: Luigi La Spada

Received: 17 May 2021

Accepted: 15 June 2021

Published: 21 June 2021

Publisher's Note: MDPI stays neutral with regard to jurisdictional claims in published maps and institutional affiliations.

Copyright: (c) 2021 by the authors. Licensee MDPI, Basel, Switzerland. This article is an open access article distributed under the terms and conditions of the Creative Commons Attribution (CC BY) license (https:/ / creativecommons.org/licenses/by/ $4.0 /)$.
School of Mechanical Engineering, Hangzhou Dianzi University, Hangzhou 310018, China; wuliqun@hdu.edu.cn (L.W.); fanyafei121@126.com (Y.F.); wanghc@hdu.edu.cn (H.W.); zln@hdu.edu.cn (L.Z.); shengyizheng@126.com (Y.S.); wyj18855034563@163.com (Y.W.)

* Correspondence: faywinter@126.com

Abstract: Aiming at the key factors affecting the quality and efficiency of high-energy in-beam machining, this paper studies the broadband acoustic focusing effect based on a discrete temperature gradient. Firstly, the basic theory and mathematical model of temperature-controlled acoustic focusing are established. Secondly, the acoustic focusing effect is achieved by combining the design of metasurfaces and discrete temperature. Then, the acoustic pressure and intensity distribution of acoustic focusing under a discrete temperature gradient are simulated and experimentally studied. The results show that the phase delay of transmission and reflection of acoustic wave covers the $2 \pi$ interval by changing the temperature in different transmission units, which provides a theoretical basis for the processing of the acoustic focusing cavity.

Keywords: metasurfaces; discrete temperature gradient; acoustic focusing; inner cavity processing

\section{Introduction}

The acoustic focusing effect [1-4] has broad application prospects in the fields of acoustic imaging, ultrasonic medical treatment [5,6], and nondestructive testing [7,8]. At present, the acoustic focusing effect is widely used, with ultrasonic therapy being the most common application. It usually involves focusing a beam to guide ultrasonic energy to a small area within a tissue area that needs treatment. In such applications, nonlinear acoustic effects and acoustic cavitation usually play an important role. The tissue temperature in the focal zone can reach $70^{\circ} \mathrm{C}$ to $90^{\circ} \mathrm{C}$ in a few seconds. With the rapid development of phononic crystals and acoustic metamaterials, a variety of different types of acoustic focusing lenses have been realized by using the negative refraction mechanism of the two structures. By gradually changing the lattice size of the phononic crystal [9], Young's modulus, element shape, and lattice constant [10], the acoustic refractive index of gradient distribution can be obtained, and a variety of gradient acoustic focusing lenses can be designed. The acoustic metasurface was first proposed in 2013. A large number of experimental studies have shown that the reflection angle and refraction angle of acoustic waves on the metasurfaces of different materials are non-zero [11-13]. Accordingly, there have been several designs of acoustic metasurfaces, and many abnormal physical phenomena have been realized, such as an abnormal Doppler effect [14-16], negative refraction of sound waves, acoustic holographic imaging, and acoustic absorption [17]. With the development of acoustic metasurface materials, the problems of extreme impedance mismatch and large thickness of acoustic lens were solved. In 2016, Ye's team realized the sound vortex phenomenon by using acoustic metasurfaces [18]. Acoustic metasurfaces have a small unit size and a large negative refractive index; hence, it is possible to design a small and ultrathin acoustic focusing lens. The basic units of the related metamaterials mainly include a Helmholtz resonator [19], cross-shaped structure [20], coiling-up space structure, and impedance matching multicomponent structure [2]. The acoustic refractive index of gradient distribution is obtained by arranging different sizes of unit structures, and then the acoustic 
focusing effect is realized [21,22]. However, the acoustic focusing performance of the metasurfaces depends on the discontinuous distribution of different unit structures; moreover, the metasurface unit is composed of at least two kinds of media, which easily lead to an acoustic impedance mismatch and a narrow working frequency band, sometimes even a single frequency, thus limiting the practical application of the acoustic focusing device.

Using a gradient temperature field can change the refractive index of medium distribution, which can effectively solve the problems of acoustic impedance matching and acoustic energy loss, realize the arbitrary control of the sound wave propagation path, and obtain a series of broadband acoustic anomalies, such as acoustic focusing [23,24], acoustic stealth [25], acoustic absorption [18], and acoustic unidirectional transmission [26]. However, in the abovementioned thermoacoustic devices, in order to obtain the temperature field with a gradient distribution, it is necessary to distribute multiple heat sources in the space, according to certain rules, which leads to the disadvantages of a large space size, irregular shape, and difficult integration of thermoacoustic devices. Therefore, the design of broadband planar ultrathin thermoacoustic devices has become a key problem to be solved urgently in this field.

For this paper, using the finite element numerical simulation method [27], acoustic waves were introduced vertically incident on the metasurfaces, and two metasurfaces of the same material were used to form a transmission unit. Different temperatures were set in the transmission unit, and the phase and transmission angle of the transmission wave [28] were controlled by the temperature, such that transmission and reflection acoustic focus could be realized. A temperature gradient was used to solve the problems of acoustic impedance matching and acoustic energy matching so as to realize the acoustic focusing effect with a wide frequency band. Accordingly, a theoretical basis for the internal processing of acoustic focusing is provided.

\section{Mathematical Model and Methods}

\subsection{Basic Theory of Thermoacoustics}

Assuming that air is an ideal fluid medium and satisfies the state of an ideal gas, the relationships among the speed of sound, density of air, and temperature $T$ are as follows [21]:

$$
\begin{aligned}
& C=\sqrt{\gamma R T / M}, \\
& \rho=p_{0} M /(R T)
\end{aligned}
$$

where $\gamma=1.4$ is the air heat capacity ratio, $M=28.97 \times 10^{-3} \mathrm{~kg} / \mathrm{mol}$ is the air molar mass, $R=8.31 \mathrm{~J} /(\mathrm{mol} \cdot \mathrm{K})$ is the universal gas constant, and $p_{0}=101.325 \mathrm{kPa}$ is the atmospheric pressure at $273 \mathrm{k}$.

\subsection{Temperature Control Focusing Theory}

In this section, different temperatures were set in the transmission unit, and the phase and transmission angle of the transmission wave were regulated by the temperature, thereby focusing the transmitted wave form. When the plane wave was incident perpendicular to the metasurfaces, the incident angle was zero. Therefore, the generalized Snell law of refraction [29] can be expressed as follows:

$$
\frac{d \varphi}{d x}=\frac{\omega}{c_{2}} \sin \theta_{4}
$$

Based on the inference of the refraction law in the generalized Snell law, it can be known that, when a plane wave is incident on a vertical metasurface at a normal angle [30], the transmission angle is not zero. According to Equation (3), the expression of the transmission angle can be obtained as follows:

$$
\theta_{4}=\arcsin \left(\frac{d \varphi}{d x} \frac{c_{2}}{\omega}\right)
$$


Since the arcsine function monotonically increases, when the temperature increases, the transmission angle increases; otherwise, it decreases. The transmission angle is changed by changing the speed of sound through temperature so as to realize the deflecting direction of the transmitted wave. The phase distribution formula of the metasurface is as follows:

$$
\varphi(y)=-\frac{\omega}{c}\left(\sqrt{y^{2}+b^{2}}-b\right)
$$

where $\varphi(\mathrm{y})$ is the phase of the acoustic wave, and $b$ is the focal length.

According to the formula, the phase of acoustic wave can be changed by changing the speed of sound, and then the phase of the acoustic wave can be controlled by changing the temperature. Upon setting $\mathrm{d} \varphi / \mathrm{d} x$ to $\pi / 14$ in Equation (4), Equation (5) dictates that the speed of sound increases with the increase of temperature. Thus, temperatures of $340 \mathrm{k}$, $420 \mathrm{k}, 530 \mathrm{k}, 650 \mathrm{k}, 850 \mathrm{k}$, and $1230 \mathrm{k}$ correspond to speeds of sound of approximately $360 \mathrm{~m} / \mathrm{s}, 372 \mathrm{~m} / \mathrm{s}, 383 \mathrm{~m} / \mathrm{s}, 398 \mathrm{~m} / \mathrm{s}, 409 \mathrm{~m} / \mathrm{s}$, and $422 \mathrm{~m} / \mathrm{s}$, respectively. When the frequency is $20 \mathrm{kHz}$, these speeds of sound introduce into Equation (4) yield transmission angles of approximately $3^{\circ}, 9^{\circ}, 15^{\circ}, 21^{\circ}, 27^{\circ}$, and $33^{\circ}$, respectively. When the corresponding coordinates are negative, $\mathrm{d} \varphi / \mathrm{dx}$ is set to $-\pi / 14$, and the speeds of sound at the same temperature can be entered into Equation (4). Since the arcsine function is an odd function, this yields transmission angles of approximately $-3^{\circ},-9^{\circ},-15^{\circ},-21^{\circ},-27^{\circ}$, and $-33^{\circ}$, respectively. It is concluded that the projection angle of the acoustic wave under the effect of temperature control shows a gradient distribution in the $y$-direction; hence, combined with Equation (5), the corresponding phase delay is $3 \pi / 2,5 \pi / 4, \pi, 3 \pi / 4, \pi / 2$, and $\pi / 4$, respectively. Through the superposition of acoustic waves, the acoustic $d$ intensity at the focal point is maximized. The schematic diagram of the temperature-controlled focusing principle is shown in Figure 1.

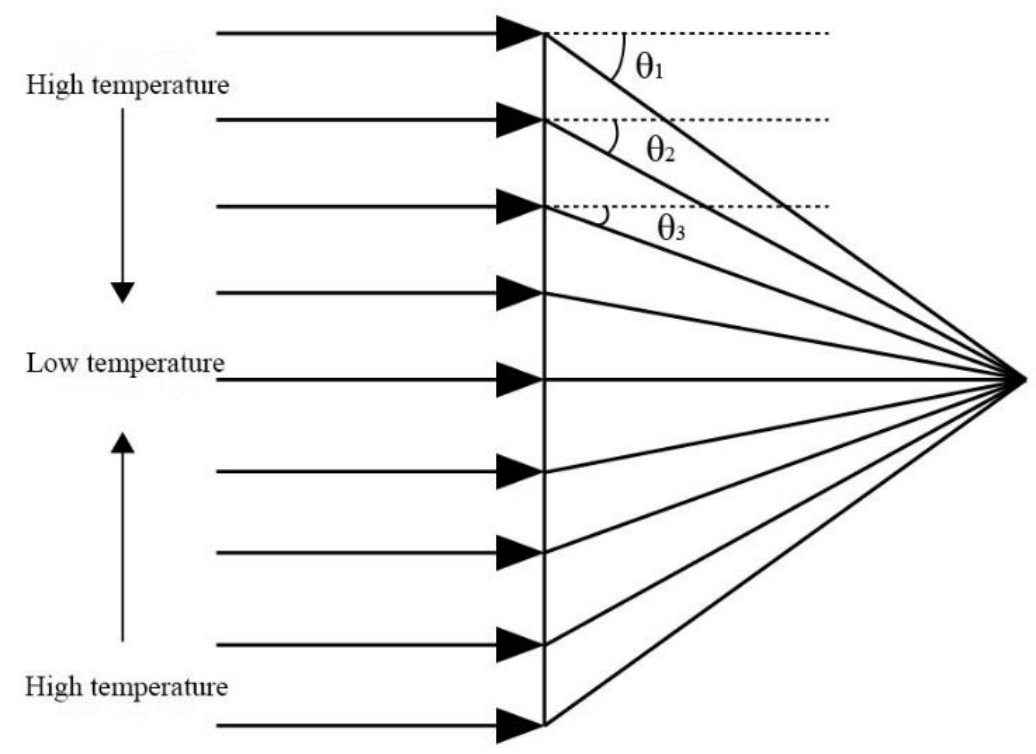

Figure 1. Schematic diagram of temperature-controlled focusing principle.

It can be seen from Figure 1 that the high-temperature area has a large acoustic wave transmission angle, a smaller phase delay, and a larger speed of sound, but the acoustic wave travels farther. The low-temperature area has a smaller acoustic wave transmission angle, a greater phase delay, and a slower speed of sound, but the distance is relatively short; thus, the acoustic waves eventually arrive at the same point in the same phase to form an acoustic wave gathering. 


\subsection{Helmholtz Equation}

Based on the equation of motion, the equation of continuity, and the equation of state, it can be concluded that the three-dimensional wave equation is as follows:

$$
\nabla^{2} p_{1}=\frac{1}{c_{0}^{2}} \frac{\partial^{2} p}{\partial t^{2}}
$$
follows:

The three-dimensional wave equation above can be obtained by Fourier transform as

$$
\nabla\left(\frac{1}{\rho_{0}} \nabla p\right)+\frac{\omega^{2}}{\rho_{0} c^{2}} p=0
$$

Substituting Equation (6) into Equation (7) gives the Helmholtz equation.

$$
\nabla\left(\frac{1}{\rho_{0}} \nabla p\right)+\frac{\omega^{2}}{\rho_{0} c^{2}} p=0
$$

\subsection{Modeling and Boundary Condition Setting}

Plane wave focusing with a discrete temperature gradient is a method combining an acoustic metasurface [31] with discrete temperature. The length of the metasurface was set to $1 \mathrm{~cm}$, the frequency was set to $20 \mathrm{kHz}$, and the speed of sound was set to $343 \mathrm{~m} / \mathrm{s}$, yielding a wavelength of approximately $2 \mathrm{~cm}$, thus meeting the design requirement of the metasurface. It can be seen from Figure 2 that the two linear metasurfaces and boundaries 4 and 5 formed a rectangular transmission unit. By setting different constant temperatures in the transmission unit, using the temperature to change the speed of sound and air density, the acoustic impedance in the transmission unit could be changed, such that the acoustic wave was abnormally transmitted. Hence, the temperature could be used to control the transmission angle and the phase of the acoustic wave, thereby focusing the transmitted acoustic wave. Firstly, the propagation model of the plane wave in a single transmission unit was established, as shown in Figure 2.

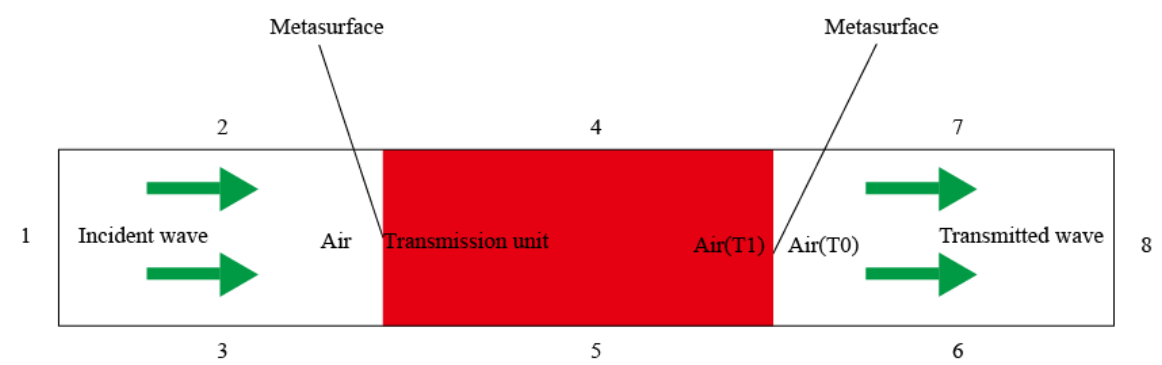

Figure 2. Schematic diagram of transmission unit.

The length of the three rectangular areas was $10 \mathrm{~cm}$ and the width was $1 \mathrm{~cm}$. The plane wave propagated along the $x$-direction, transmitted into the middle constant-temperature transmission unit, and then transmitted out of the constant-temperature transmission unit. Boundaries 1 and 8 were set as plane wave radiation, and an incident pressure field with a size of 1 Pa was added to boundary 1 , representing the sound pressure amplitude of incident sound waves. The materials were all air. Temperatures in the transmission unit were set to $430 \mathrm{~K}, 530 \mathrm{~K}, 650 \mathrm{~K}, 760 \mathrm{~K}, 850 \mathrm{~K}, 970 \mathrm{~K}$, and $1190 \mathrm{~K}$.

\section{Numerical Results and Discussion}

3.1. Acoustic Pressure and Intensity Distribution of Transmitted Wave Focusing under Discrete Temperature Gradient

Based on the parameters and boundary conditions set in Section 2.4, the acoustic pressure distribution of the plane wave in the transmission unit at different temperatures was simulated, using the multi-physics simulation software COMSOL, as shown in Figure 3. 


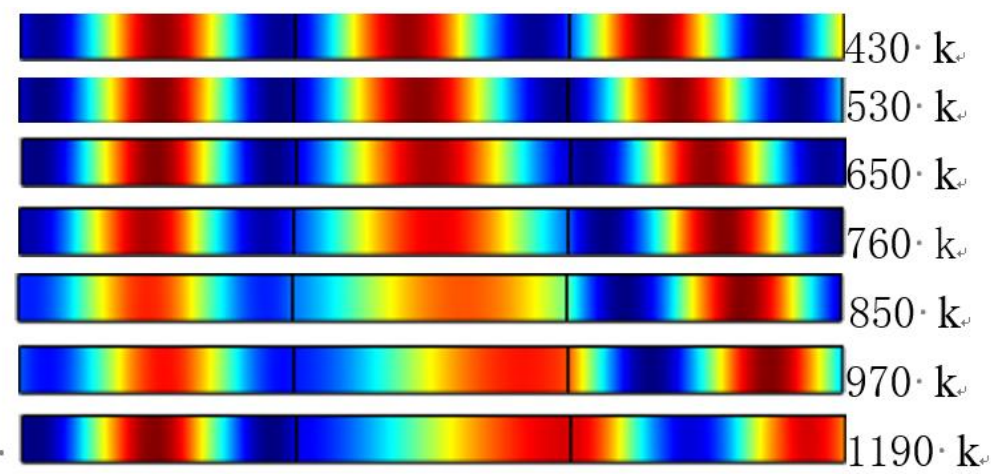

Figure 3. Acoustic pressure distribution of transmission unit at different temperatures.

It can be seen from the numerical simulation results that when the acoustic wave was transmitted into the constant-temperature transmission unit, the sound of speed increased with the increase in temperature; furthermore, when the frequency was constant, the wavelength of the acoustic wave increased. According to the wavelength formula and the sound of speed in air being $343 \mathrm{~m} / \mathrm{s}$, the distance of the acoustic wave before transmission was greater than one wavelength, but the distance of the acoustic wave in the transmission unit with constant temperature was obviously less than one wavelength. It can be concluded that the same frequency of the acoustic source, when launched at the same time, would not result in the same distance, reflecting the influence of different temperatures on the acoustic phase.

Based on the propagation law of the plane wave in the transmission units at different temperatures, the transmission waves were focused by arranging the transmission units with different temperatures. The model is shown in Figure 4.

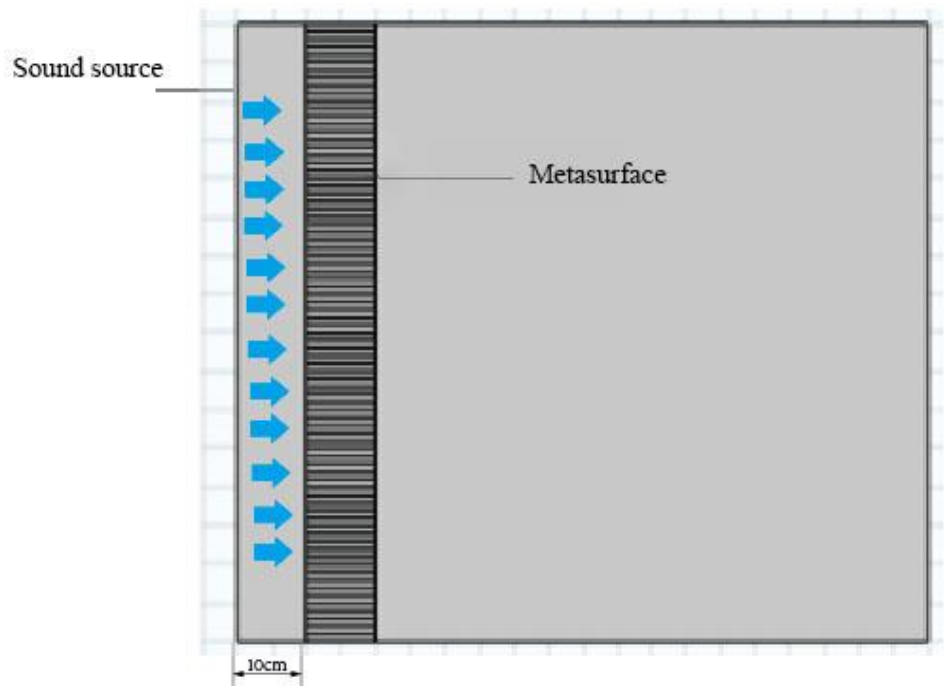

Figure 4. Distribution of the metasurface on the $y$-axis.

Taking $y=80 \mathrm{~cm}$, the speed of sound at different temperatures was introduced into Equations (1) and (5), along with the frequencies of acoustic waves and a focal length of $30 \mathrm{~cm}$, to obtain the phase change corresponding to different temperatures. Temperatures of $430 \mathrm{k}, 530 \mathrm{k}, 650 \mathrm{k}, 760 \mathrm{k}, 850 \mathrm{k}, 970 \mathrm{k}$ and $1190 \mathrm{k}$ corresponded to phase delays of $7 \pi / 4,3 \pi / 2,5 / 4 \pi, \pi, 3 \pi / 4, \pi / 2$, and $\pi / 4$, respectively, as shown in Figure 5 . It can be seen that the transmission wave produced a phase delay when the acoustic wave passed through the thermoacoustic phase control unit. 


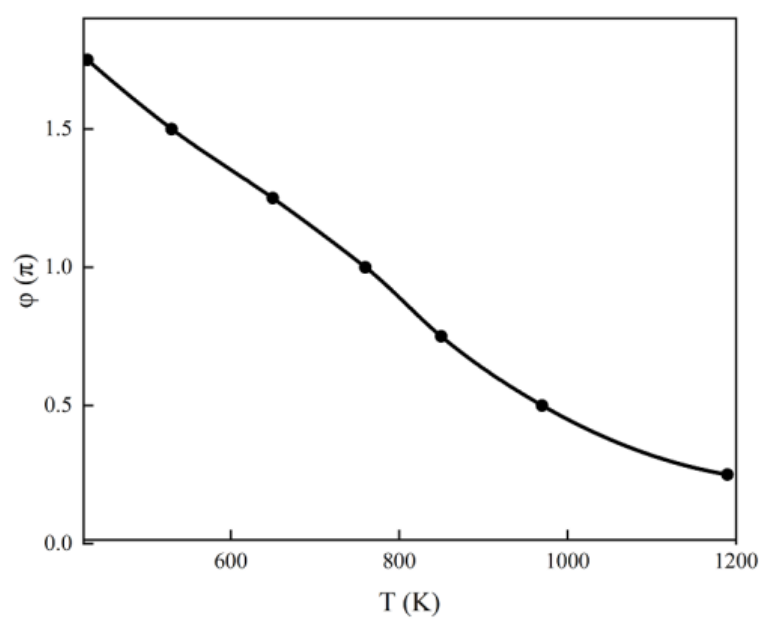

Figure 5. The delay of acoustic phase corresponding to temperature.

The function of Equation (5) was drawn, using the Origin drawing software. Seven kinds of transmission units with different temperatures corresponded to seven kinds of phase delays of acoustic waves. The distribution of transmission units is shown in Figure 6.

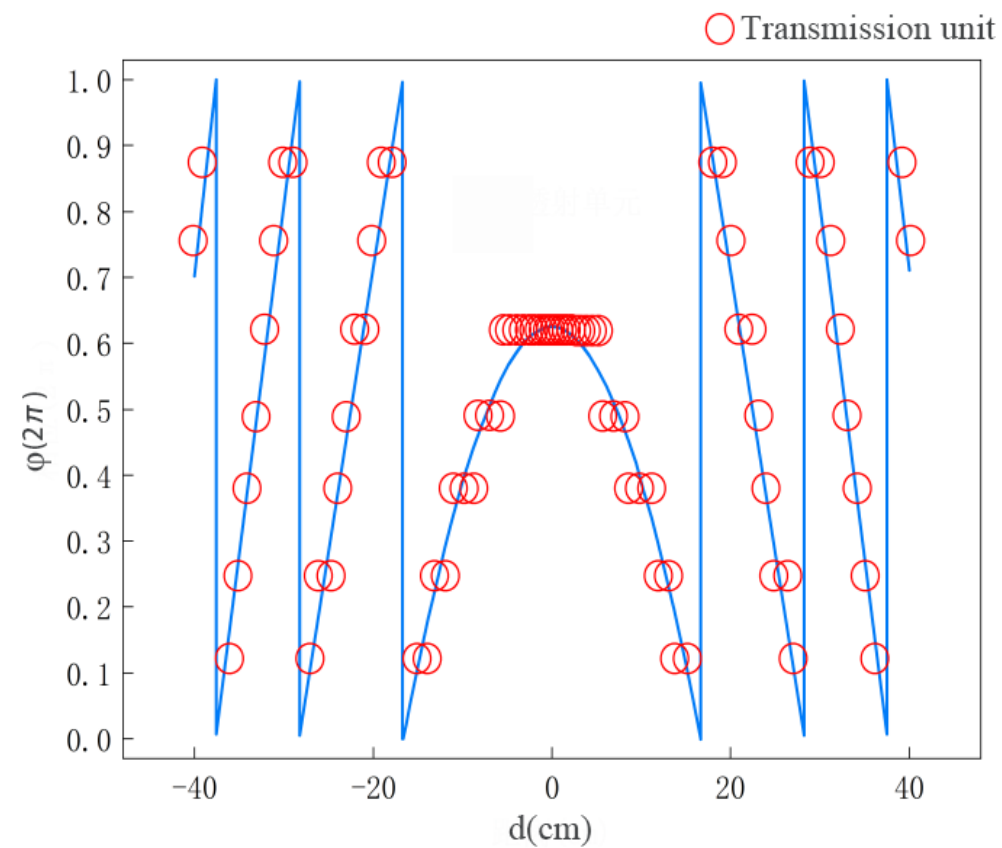

Figure 6. Distribution of transmission units in the $y$-direction.

As can be seen in Figure 6, from $y=40 \mathrm{~cm}$ to $y=0 \mathrm{~cm}$, the fluctuating temperature led to the transmission angle having a positive value before gradually decreasing. From $y=$ $0 \mathrm{~cm}$ to $y=-40 \mathrm{~cm}$, the fluctuating temperature led to the opposite effect. On the $y$-axis, the transmission angle at both ends was larger, and the transmission angle in the middle was smaller, forming a gradient distribution of transmission angle on the $y$-axis.

By establishing a model in COMSOL software and setting the parameters and boundary conditions, the acoustic pressure and acoustic intensity distribution of the transmitted wave focusing under a discrete temperature gradient could be obtained, as shown in Figures 7 and 8. 


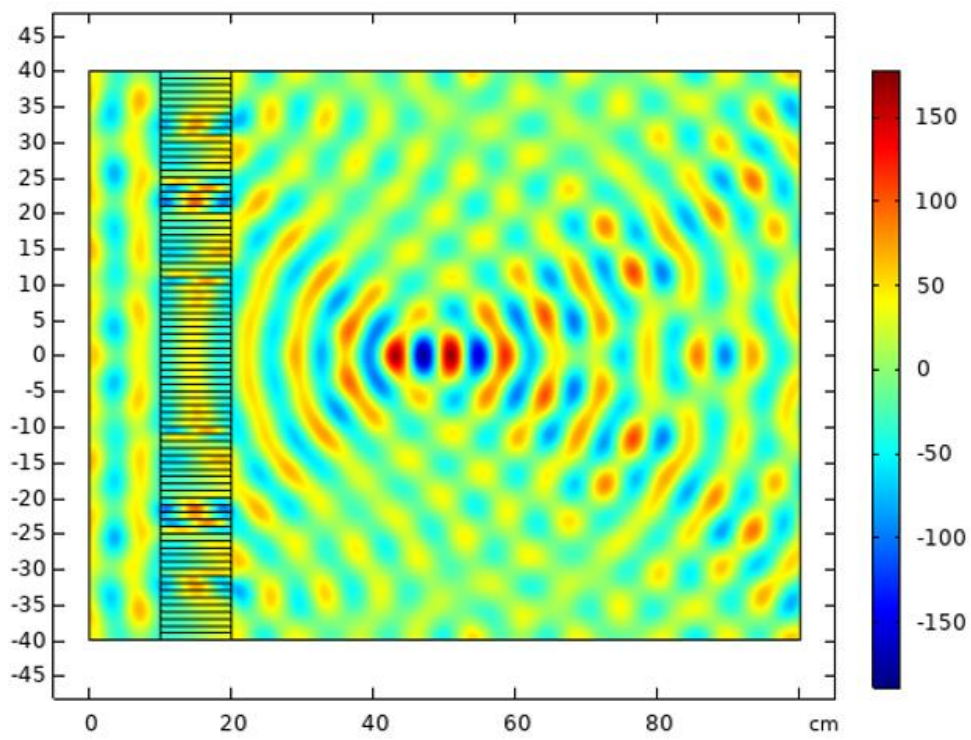

Figure 7. Spatial distribution of acoustic pressure field through focusing lens at $20 \mathrm{kHz}$.

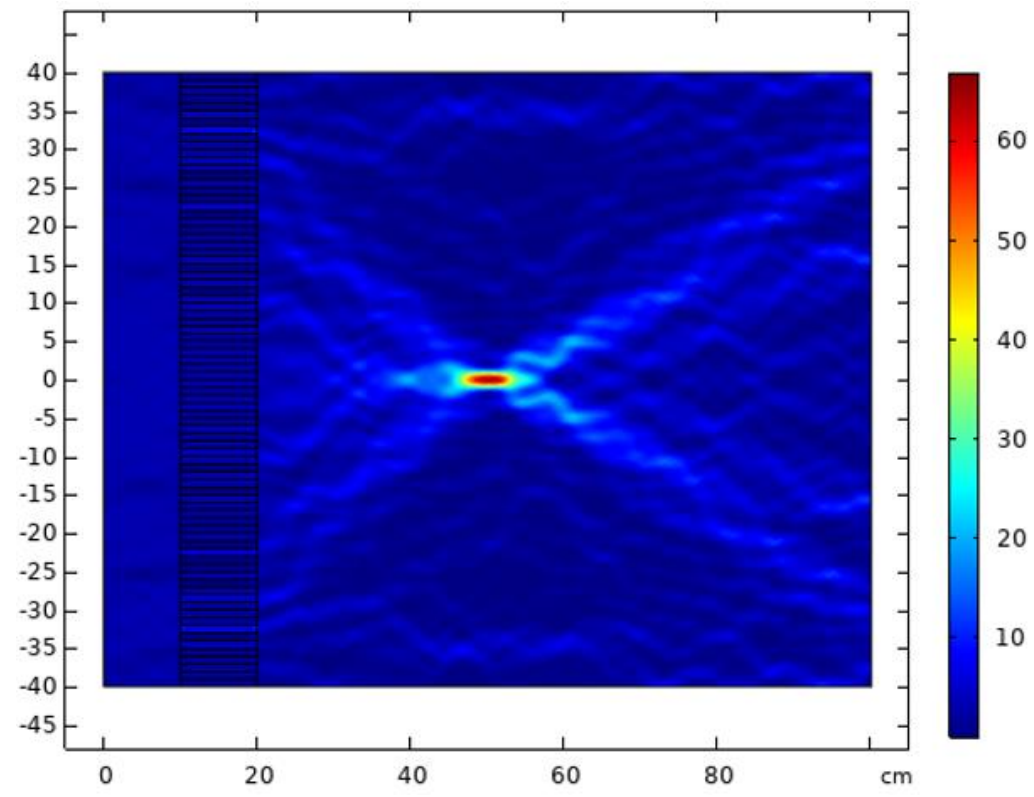

Figure 8. Spatial distribution of acoustic intensity field through focusing lens at $20 \mathrm{kHz}$.

From Figure 8, it can be concluded that the focal length was about $30 \mathrm{~cm}$, thus verifying the previously set parameters. Compared with the numerical simulation, the acoustic focusing model obtained by combining the discrete temperature gradient and metasurface showed great advantages. It facilitated the focusing of the acoustic wave [32,33], as well as expanded its formation during inner cavity processing.

In order to overcome the limitation of a single-sound source frequency on the acoustic focusing effect, temperature control was used to solve the problem of acoustic impedance matching so as to achieve a wideband acoustic focusing effect. First, frequencies of $28 \mathrm{kHz}$, $35 \mathrm{kHz}, 40 \mathrm{kHz}, 45 \mathrm{kHz}, 50 \mathrm{kHz}$ and $55 \mathrm{kHz}$ were selected, corresponding to temperatures of $530 \mathrm{k}, 650 \mathrm{k}, 850 \mathrm{k}, 990 \mathrm{k}, 1120 \mathrm{k}$ and $1260 \mathrm{k}$ respectively. Then, a numerical simulation was used to evaluate the effect of different plane wave frequencies on the focusing. The simulation results are shown in Figure 9. 


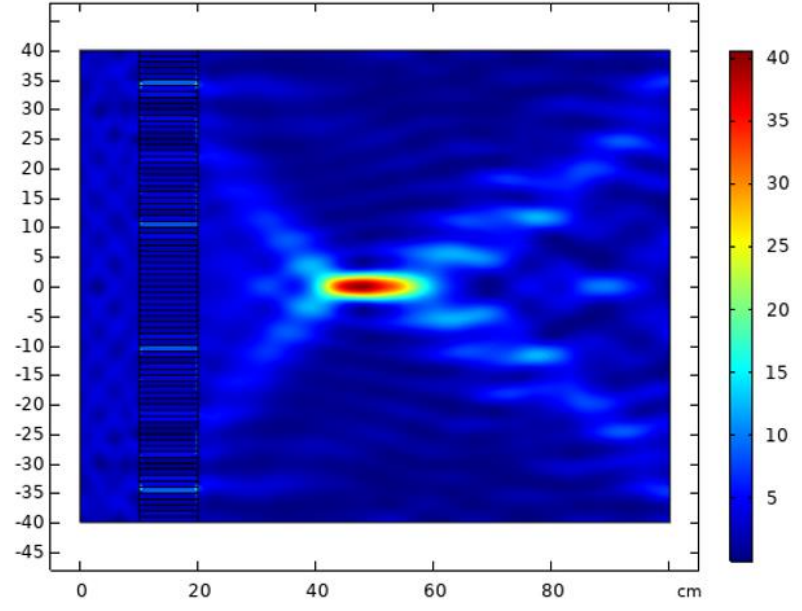

(a)

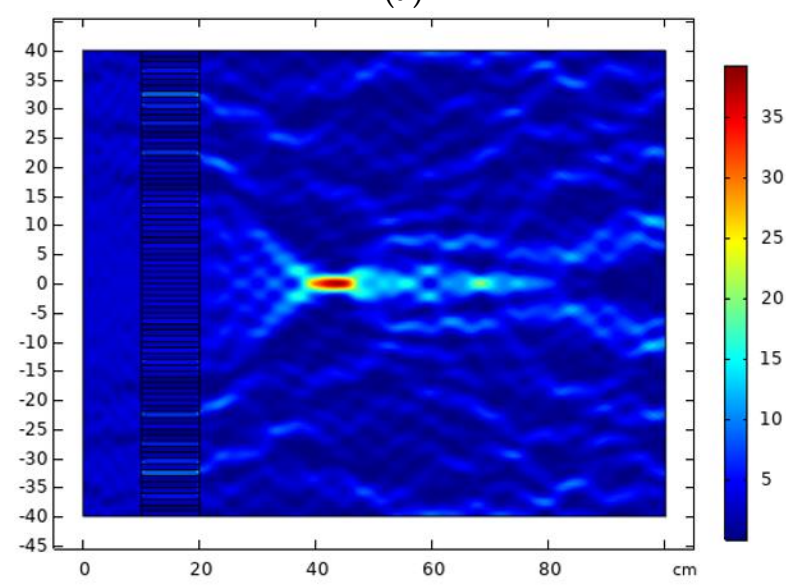

(c)

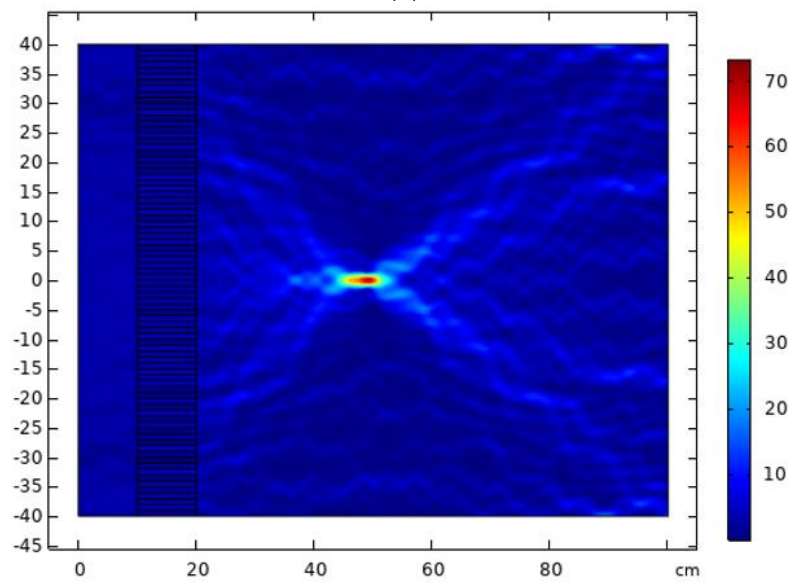

(e)

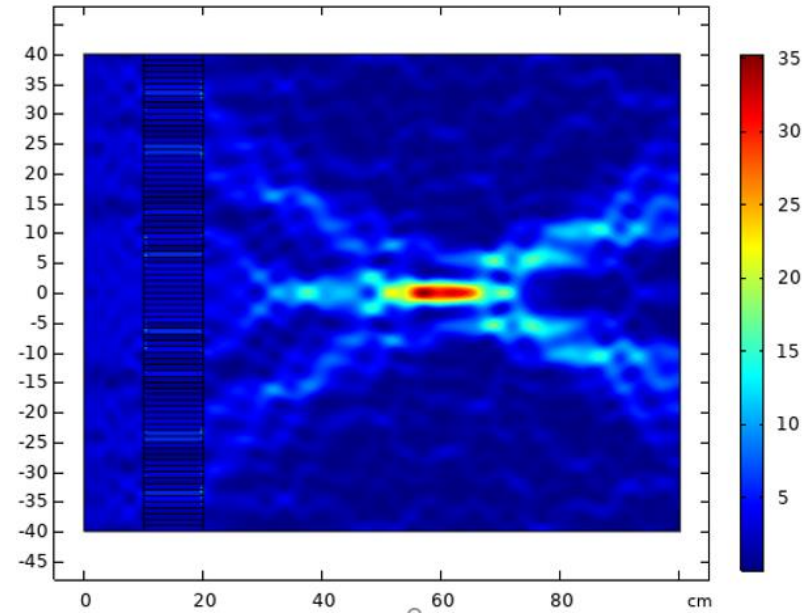

(b)

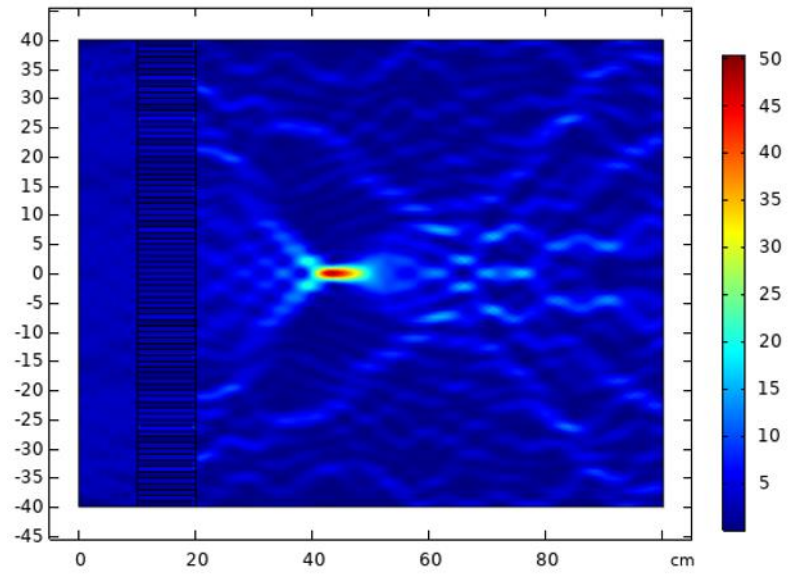

(d)

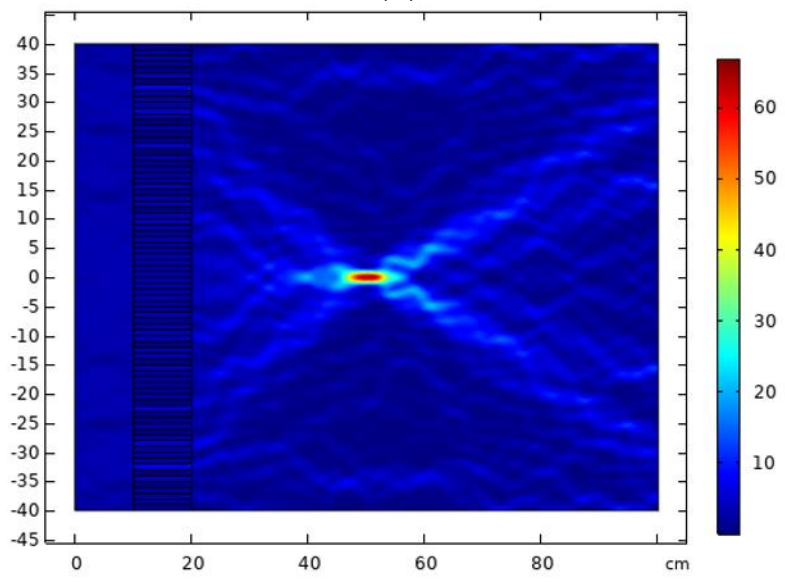

(f)

Figure 9. Spatial distributions of acoustic intensity field through focusing lens at (a) $28 \mathrm{kHz}$, (b) $35 \mathrm{kHz}$, (c) $40 \mathrm{kHz}$, (d) $45 \mathrm{kHz}$, (e) $50 \mathrm{kHz}$, and (f) $55 \mathrm{kHz}$.

As can be seen from the above figure, in the frequency band from $20 \mathrm{kHz}$ to $35 \mathrm{kHz}$, the acoustic intensity gradually decreased with the increase in frequency. However, the acoustic intensity gradually increased in the $40 \mathrm{kHz}$ to $55 \mathrm{kHz}$ frequency band. The main reason is that, when the transmitted waves of different phases arrived at the same point, the phase difference between them was neither zero nor an even multiple of $\pi$. When the frequency of the plane wave increased, according to Equation (4), the transmission angle of the acoustic wave changed by changing the frequency of the sound wave, consequently 
changing the position of the focal point. Therefore, the internal machining of different positions could be realized by changing the frequency.

\subsection{Influence of Metasurfaces at Different Positions on Focusing}

The transmission unit array was previously placed $10 \mathrm{~cm}$ away from the sound source in the $x$-direction. This time, the transmission unit array was placed $0 \mathrm{~cm}$ away from the sound source in the $x$-direction, and the propagation of plane waves in transmission units at different temperatures was studied. Two plane waves with different frequencies were randomly selected for COMSOL simulation. The simulation results are shown in Figure 10.

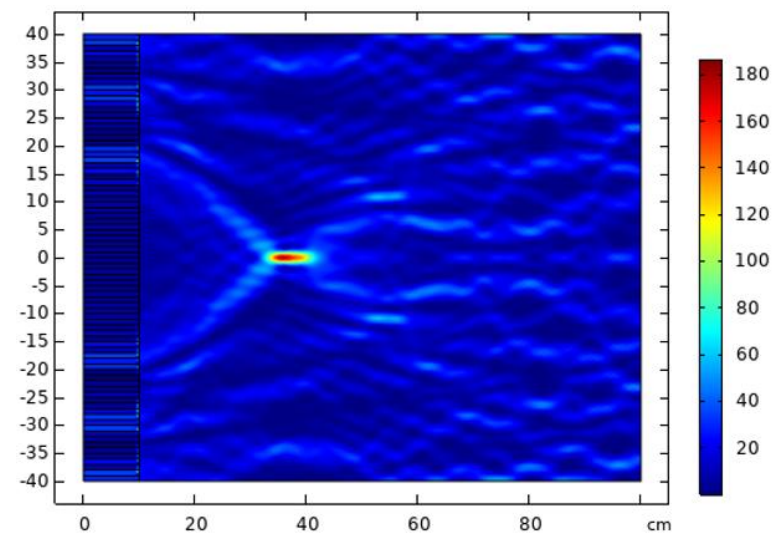

(a)

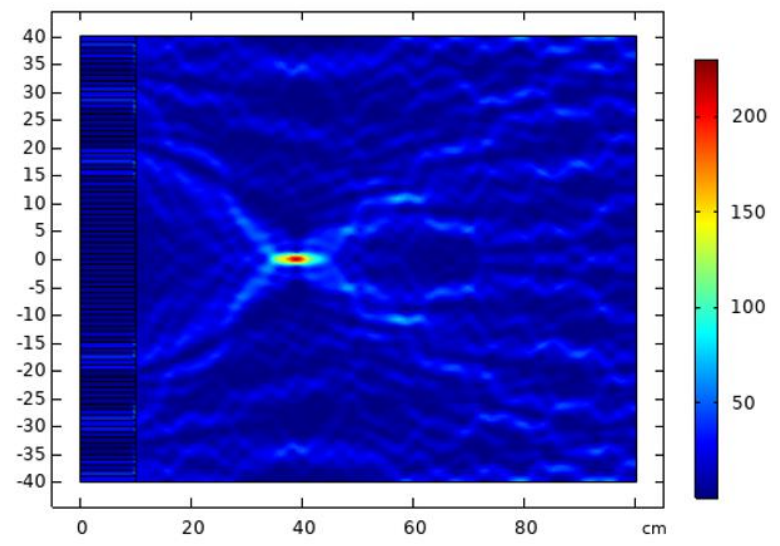

(b)

Figure 10. Spatial distributions of acoustic intensity field through focusing lens at (a) $28 \mathrm{kHz}$ and (b) $35 \mathrm{kHz}$.

At the same frequency, when the transmission unit array was $0 \mathrm{~cm}$ away from the sound source in the $x$-direction, the sound intensity at the focus point was greater. The main reason is that when the transmission unit array was $10 \mathrm{~cm}$ away from the sound source in the $x$-direction, the sound wave was transmitted twice. However, by changing the position of the transmission element array, the gradient distribution of the transmission angle was still formed in the $y$-axis direction; thus, the sound wave could still form a broadband focus, whose position could be adjusted by changing the position of the transmission element array. In the process of acoustic focusing, the array position could be changed to control the focus position.

\subsection{Focusing of Reflected Waves under Discrete Temperature Gradients}

First, a plane wave propagation model was established in a single reflection unit as shown in Figure 11.

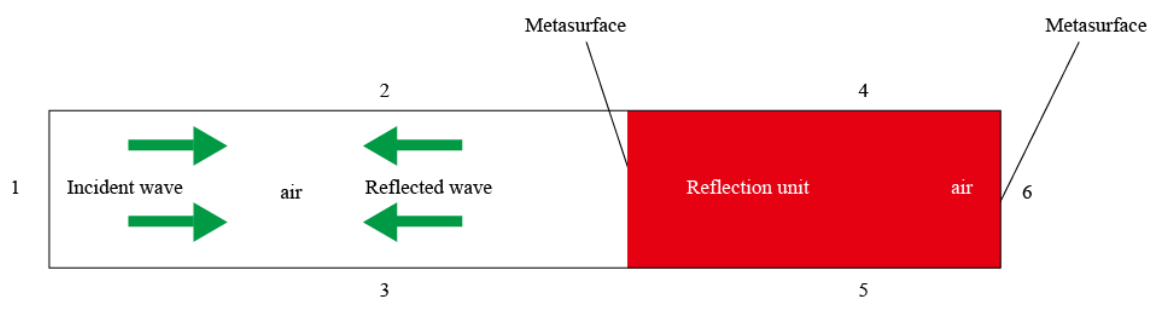

Figure 11. Schematic diagram of plane wave propagation in reflection unit.

It can be seen from Figure 11 that the plane wave propagates along the $x$-direction, before being transmitted into the reflecting unit. Upon meeting the rigid boundary, most of it is reflected back, before finally being transmitted from the reflecting unit to the air at normal temperature. 
Boundaries 1-5 were all set as plane wave radiation. An incident pressure field was additionally added to boundary 1 to represent the sound source. The amplitude of sound pressure was $1 \mathrm{~Pa}$. Boundary 6 was set as a rigid, hard sound field boundary. The acoustic source was a plane wave with a frequency of $20 \mathrm{kHz}$, and the medium was air. Seven reflection units with different temperatures were adopted $(460 \mathrm{k}, 590 \mathrm{k}, 730 \mathrm{k}, 850 \mathrm{k}, 990 \mathrm{k}$, $1120 \mathrm{k}$, and $1260 \mathrm{k}$ ). The simulation results are shown in Figure 12.

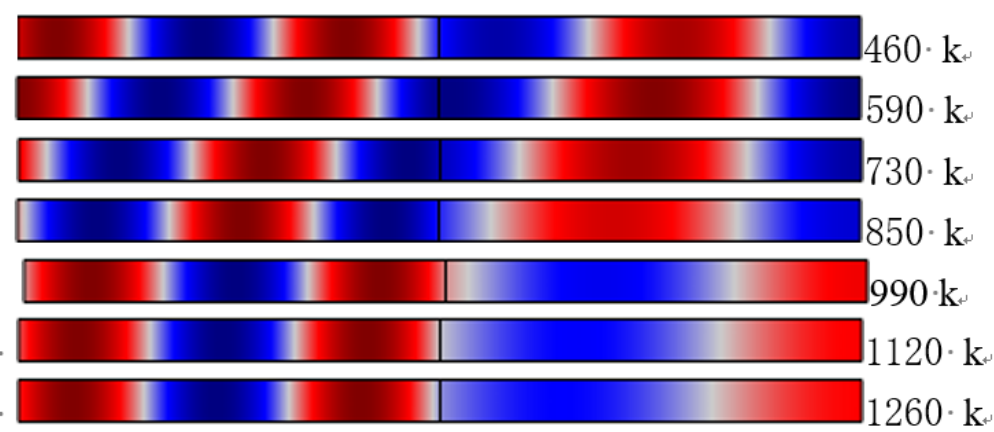

Figure 12. Acoustic pressure distribution of plane wave in seven reflection units with different temperatures.

According to the numerical simulation and Equation (5), it can be seen that the speed of sound could be transformed into the phase difference of acoustic waves, and the temperature changed the phase of sound waves by changing the speed of sound, thus controlling the speed of sound wave propagation.

Consider a focal length of $25 \mathrm{~cm}$, y value of $80 \mathrm{~cm}$, frequency of $20 \mathrm{kHz}$, and sound of speed corresponding to $460 \mathrm{k}, 590 \mathrm{k}, 730 \mathrm{k}, 850 \mathrm{k}, 990 \mathrm{k}, 1120 \mathrm{k}$ and $1260 \mathrm{k}$. Introducing these values into Equations (1) and (5) yields the phase delays corresponding to different temperatures, as shown in Figure 13.

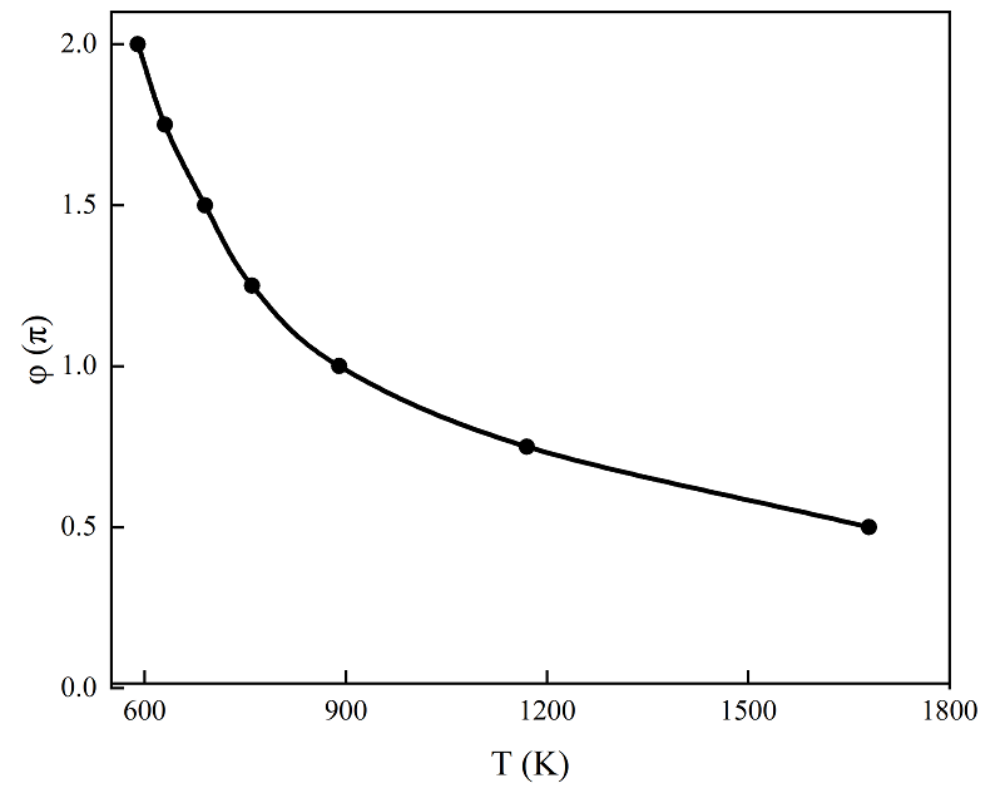

Figure 13. Phase delay corresponding to different temperatures.

The reflected wave focusing model is shown in Figure 14. 


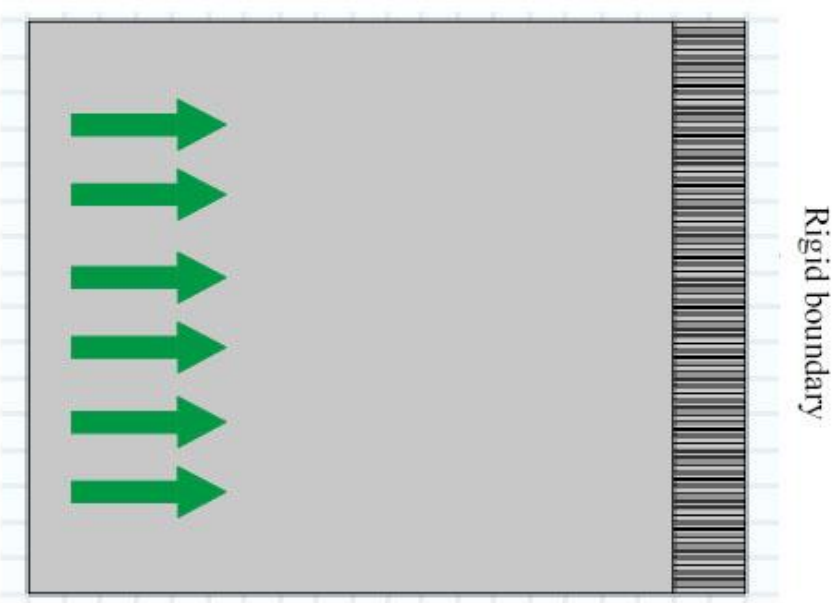

Figure 14. Reflection wave focusing model diagram.

Combined with Equation (4), it can be further obtained that the temperature changed the speed of sound, thus changing the deflection angle of the sound wave. When the sound wave propagated to the rigid boundary, most of it was reflected back. When transmitted to the air at normal temperature, the transmission angle of the $y$-axis presented a gradient distribution, thereby focusing the acoustic wave forms. When the frequency was set to $20 \mathrm{kHz}$, the acoustic intensity distribution of the focused reflection wave in the simulation was as shown in Figure 15.

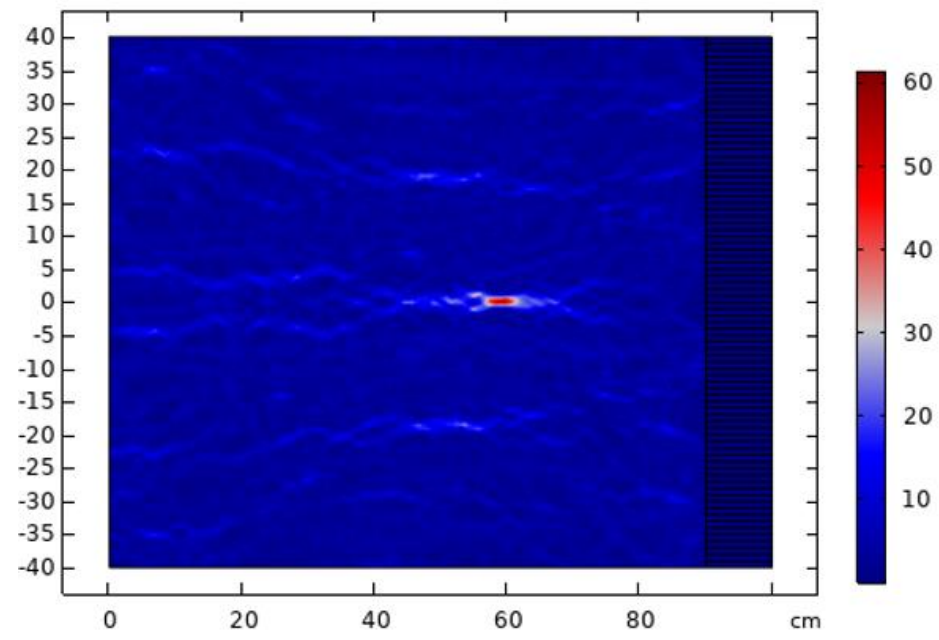

Figure 15. Spatial distribution of acoustic intensity field through focusing lens at $20 \mathrm{kHz}$.

It can be seen from Figure 15 that the focal length was about $25 \mathrm{~cm}$, which agrees with the previously set focal length value. Compared with the acoustic intensity at the focal point formed by the transmitted wave, the acoustic intensity at the focal point of the reflected wave was significantly smaller [34]. Because the focus formed by the reflected wave was transmitted three times, there was some loss in the reflection of the sound wave at the rigid boundary.

Furthermore, the influence of different frequencies on the acoustic intensity at the focal point was numerically simulated by a frequency sweep in COMSOL, and the simulation results are shown in Figure 16. 


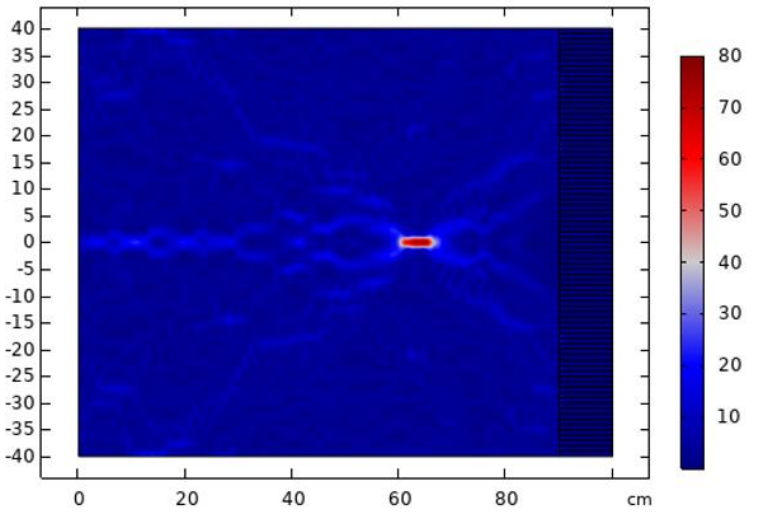

(a)

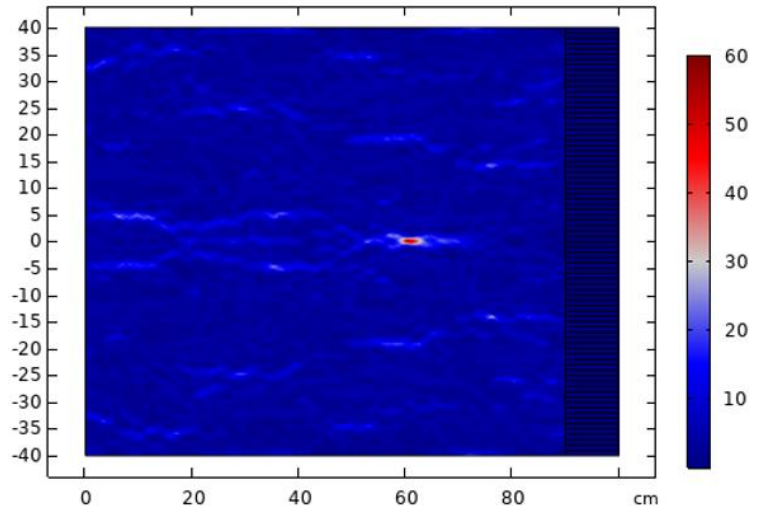

(c)

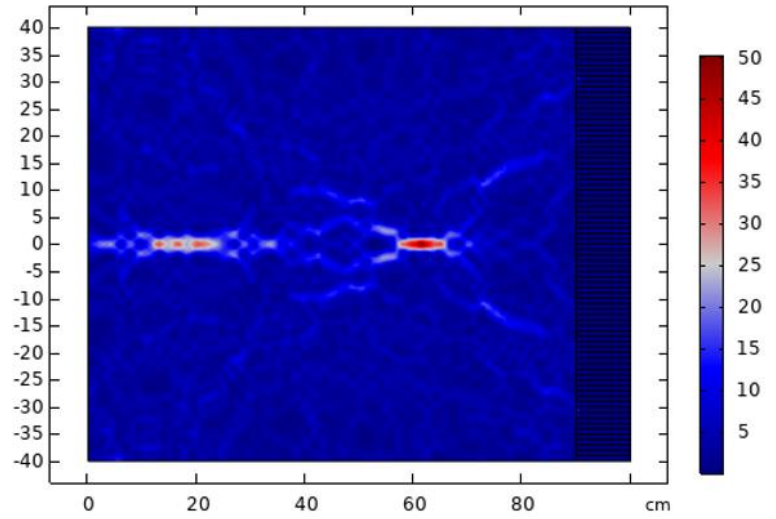

(b)

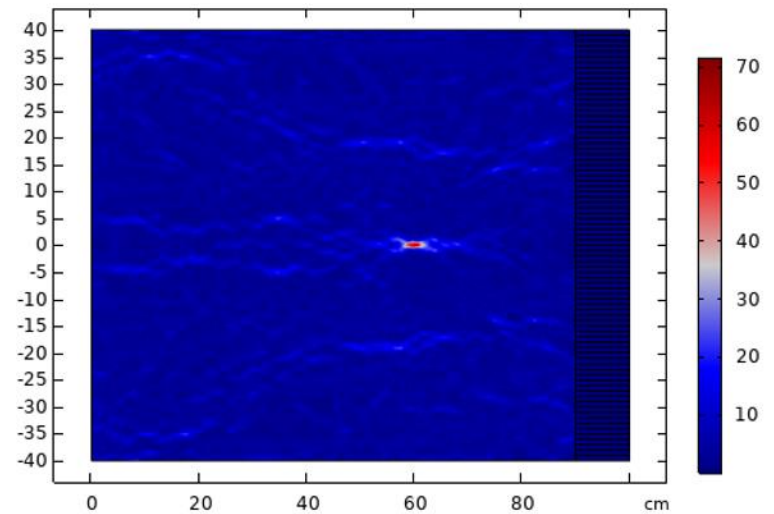

(d)

Figure 16. Spatial distributions of acoustic intensity field through focusing lens at (a) $28 \mathrm{kHz},(\mathbf{b}) 35 \mathrm{kHz}$, (c) $40 \mathrm{kHz}$, and (d) $45 \mathrm{kHz}$.

It can be seen from Figure 16 that the acoustic intensity changed non-linearly as the frequency increased. This is because different frequencies resulted in different phases of the reflected waves, and the phase difference of the reflected waves of different phases at the focus point was not zero. As a result, the acoustic intensity at the focus point was in the $28 \mathrm{kHz}$ to $35 \mathrm{kHz}$ frequency band, and the acoustic intensity gradually decreased, whereas the acoustic intensity gradually increased between $40 \mathrm{kHz}$ and $45 \mathrm{kHz}$. However, with the increase in frequency of acoustic waves, the focusing effect could not be formed upon reaching $50 \mathrm{kHz}$. The simulation results are shown in Figure 17.

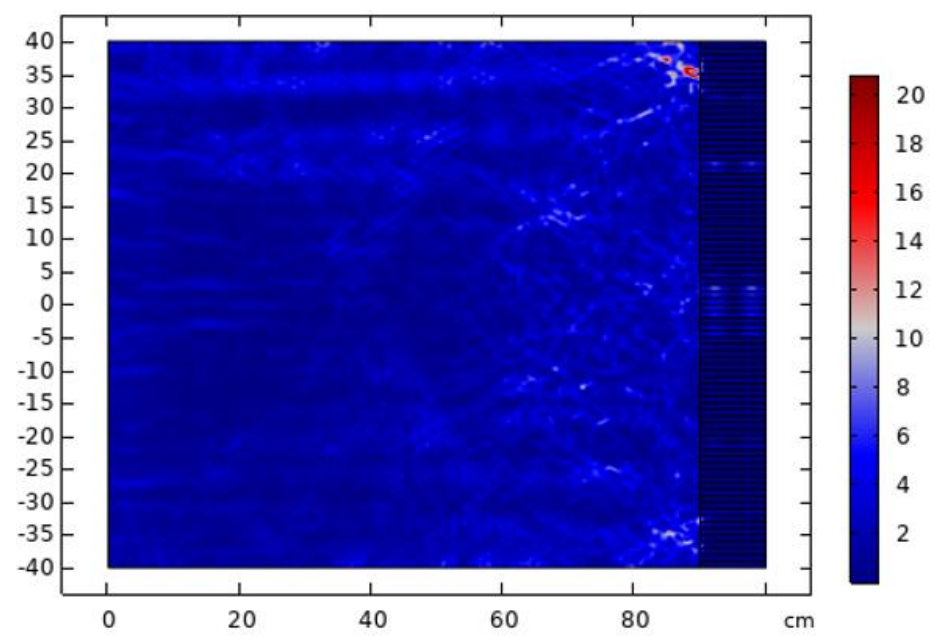

Figure 17. Spatial distribution of acoustic intensity field through focusing lens at $50 \mathrm{kHz}$. 
The effect of different frequencies on the focused acoustic intensity of the transmitted wave and reflected wave was plotted, as shown in Figure 18.

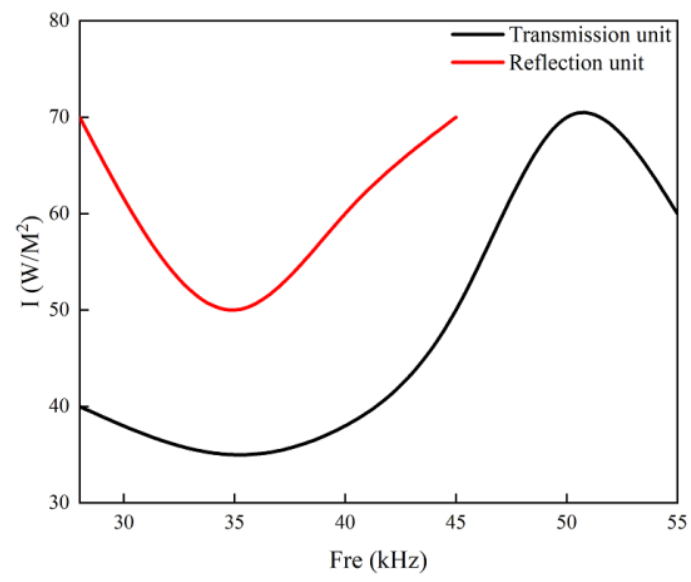

Figure 18. The influence of frequency on the sound intensity at the focal point of transmitted and reflected waves.

From Figure 18, it can be concluded that both the reflected wave and the transmitted wave can focus on a wider frequency band, but the focused frequency band formed by the reflected wave is narrower than that of the transmitted wave, and it cannot focus after $50 \mathrm{kHz}$. According to Equation (5), the focus of the reflected wave is closely related to the focal length and preset initial frequency. When it is not at the set focusing frequency, focus occurs because the deflection direction of the wave is crossed by the temperature control effect. When it reaches a certain frequency, it is impossible to focus because the difference between the focusing frequency and the initial one is too large, and the wave direction changes too much. Therefore, in order to refocus the reflected wave after $50 \mathrm{kHz}$, it is necessary to change the position of the initial focal length, the temperature, and the model width in order to regain the acoustic focusing phase distribution. It is concluded that under the action of temperature control, the reflection angle of sound wave is distributed in gradient in $y$ direction, thus reforming the focusing effect. COMSOL numerical simulation software was applied to simulate the refocusing results of reflected waves with focal length $b=20 \mathrm{~cm}, y=80 \mathrm{~cm}$, and frequency $=50 \mathrm{kHz}$; the results are shown in Figure 19.

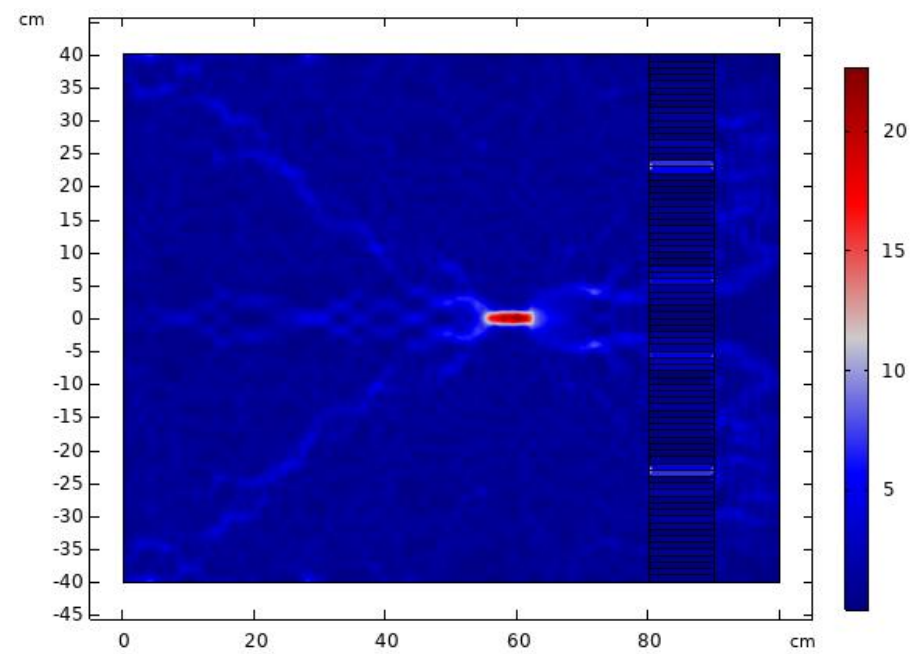

Figure 19. Spatial distribution of acoustic intensity field through focusing lens at $50 \mathrm{kHz}$. 


\subsection{Acoustic Transmission and Reflection Focusing Model}

From the perspective of ultrasonic internal processing, it can be obtained from the numerical simulation results that the intensity of the acoustic wave at the focus point is slightly insufficient for inducing the generation of cavitation bubbles by cavitation nuclei $[35,36]$. Therefore, by designing a new type of acoustic wave combining the transmitted wave focusing model and the reflected wave focusing model, the purpose is to enhance the sound intensity at the sound wave focusing point. The focusing intensity generated by operating the acoustic focusing model can be greater than the acoustic cavitation threshold, thus inducing the generation of cavitation bubbles by cavitation nuclei and then achieving the purpose of ultrasonic internal processing. The simulation results of the acoustic wave transmission and reflection focusing model are shown in Figure 20.

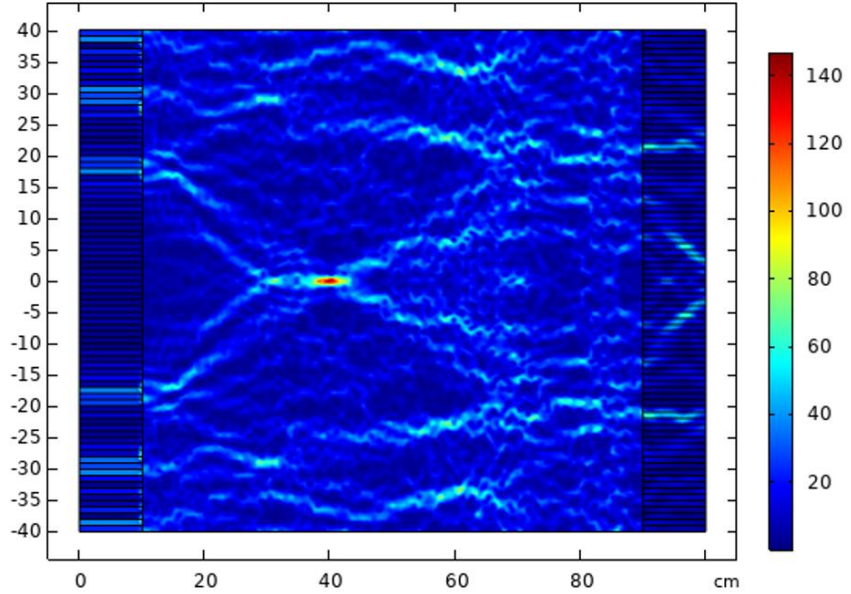

(a)

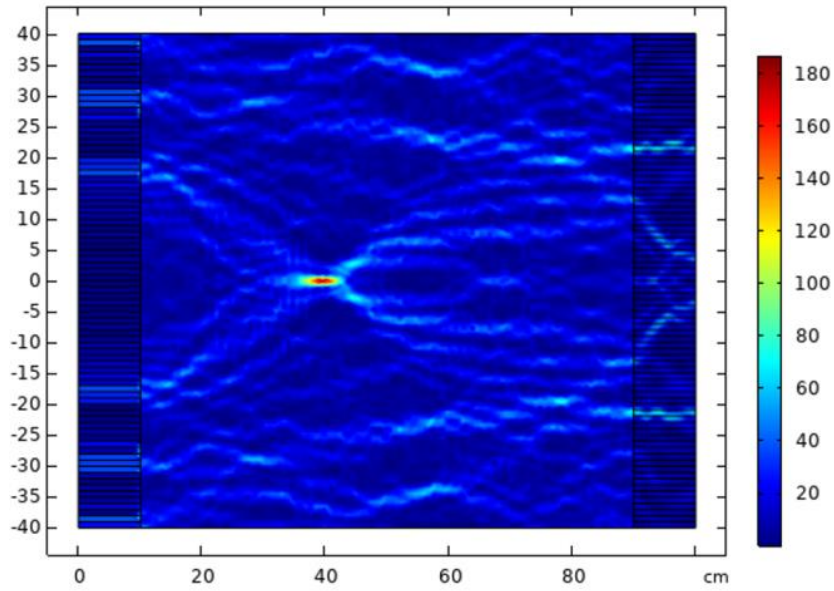

(b)

Figure 20. Spatial distributions of acoustic intensity field through focusing lens at (a) $28 \mathrm{kHz}$ and (b) $35 \mathrm{kHz}$.

\section{Conclusions}

On the basis of the relationship between the speed of sound and temperature of air, combined with the generalized Snell law, a new thermoacoustic phase control unit was designed in this paper by combining the metasurfaces and discrete temperature. The acoustic focusing theory, based on the combination of the metasurfaces and discrete temperature, was subsequently verified. The numerical simulation results showed that the acoustic wave was focused on a wide frequency range from $20 \mathrm{kHz}$ to $55 \mathrm{kHz}$, thereby providing a wide frequency band for the internal processing of acoustic focusing. The whole $2 \pi$ interval was covered by the transmission and reflection phase delay of the acoustic wave. The results showed that the focusing lens designed in this paper has the advantages of wideband, high focusing, and higher robustness properties. It is of guiding significance for acoustic focusing internal processing.

Author Contributions: Conceptualization, L.W.; methodology, L.W., H.W. and L.Z.; software, Y.F.; validation, Y.S., Y.F. and Y.W. (Yajing Wang); formal analysis, L.W.; investigation, Y.F.; resources, L.W. and Y.W. (Yaxing Wang); data curation, H.W. and L.Z.; writing-original draft preparation, L.W. and Y.F.; writing-review and editing, Y.F. and Y.S.; supervision, Y.W. (Yaxing Wang); funding acquisition, H.W., L.W. and L.Z. All authors have read and agreed to the published version of the manuscript.

Funding: This work is supported by National Natural Science Foundation of China under Grant No. 51775154 and 11902107 and ZheJiang Provincial Natural Science Foundation of China under Grant No. LY21F040005.

Institutional Review Board Statement: Not applicable.

Informed Consent Statement: Not applicable. 
Data Availability Statement: Data sharing is not applicable to this article.

Conflicts of Interest: The authors declare no conflict of interest.

\section{References}

1. Xia, J.; Sun, H.; Cheng, Q.; Xu, Z.; Chen, H.; Yuan, S.; Zhang, S.; Ge, Y.; Guan, Y. Theoretical and experimental verification of acoustic focusing in metal cylinder structure. Appl. Phys. Express 2016, 9, 57301. [CrossRef]

2. Al Jahdali, R.; Wu, Y. High transmission acoustic focusing by impedance-matched acoustic meta-surfaces. Appl. Phys. Lett. 2016, 108, 31902. [CrossRef]

3. Guan, Y.; Sun, H.; Liu, S.; Yuan, S.; Xia, J.; Ge, Y. Acoustic focusing through two layer annuluses in air. Chin. Phys. B 2016, 25, 182-188. [CrossRef]

4. Xia, J.; Sun, H. Acoustic focusing by metal circular ring structure. Appl. Phys. Lett. 2015, 106, 63505. [CrossRef]

5. Gu, Y.; Cheng, Y.; Liu, X. Acoustic planar hyperlens based on anisotropic density-near-zero metamaterials. Appl. Phys. Lett. 2015, 107, 133503. [CrossRef]

6. Zhao, J.; Ye, H.; Huang, K.; Chen, Z.N.; Li, B.; Qiu, C. Manipulation of acoustic focusing with an active and configurable planar metasurface transducer. Sci. Rep. 2014, 4, 6257. [CrossRef] [PubMed]

7. Chen, Y.; Logan, P.; Avitabile, P.; Dodson, J. Non-Model Based Expansion from Limited Points to an Augmented Set of Points Using Chebyshev Polynomials. Exp. Tech. 2019, 43, 521-543. [CrossRef]

8. Zhu, Z.; Luo, S.; Feng, Q.; Chen, Y.; Wang, F.; Jiang, L. A hybrid DIC-EFG method for strain field characterization and stress intensity factor evaluation of a fatigue crack. Measurement 2020, 154, 107498. [CrossRef]

9. Martin, T.P.; Layman, C.N.; Moore, K.M.; Orris, G.J. Elastic shells with high-contrast material properties as acoustic metamaterial components. Phys. Rev. B Condens. Matter Mater. Phys. 2012, 85, 161103. [CrossRef]

10. Torrent, D.; Sánchez-Dehesa, J. Acoustic metamaterials for new two-dimensional sonic devices. New J. Phys. 2007, 9, 323. [CrossRef]

11. Zhao, J.; Li, B.; Chen, Z.; Qiu, C. Manipulating Acoustic Wavefront by Inhomogeneous Impedance and Steerable Extraordinary Reflection. Sci. Rep. 2013, 3, 2537. [CrossRef] [PubMed]

12. Zhao, J.; Li, B.; Chen, Z.N.; Qiu, C. Redirection of sound waves using acoustic metasurface. Appl. Phys. Lett. 2013, 103, 151604. [CrossRef]

13. Qian, J.; Wang, Y.; Yuan, S.; Sun, H.; Liu, X. Reflected acoustic wavefront manipulation by an ultrathin metasurface based on three-dimensional generalized Snell's law. Appl. Phys. Express 2019, 12, 94001. [CrossRef]

14. Liu, S.; Luo, C.; Zhai, S.; Chen, H.; Zhao, X. Inverse Doppler effect of acoustic metamaterial with negative mass density. Acta Phys. Sin. Ch. Ed. 2017.

15. Chen, J.; Wang, Y.; Jia, B.; Geng, T.; Li, X.; Feng, L.; Qian, W.; Liang, B.; Zhang, X.; Gu, M.; et al. Observation of the inverse Doppler effect in negative-index materials at optical frequencies. Nat. Photonics 2011, 5, 239-242. [CrossRef]

16. Kumar, S.; Lee, H. The Present and Future Role of Acoustic Metamaterials for Architectural and Urban Noise Mitigations. Acoustics 2019, 1, 35. [CrossRef]

17. Zhai, S.; Wang, Y.; Zhao, X. A kind of tunable acoustic metamaterial for low frequency absorption. Acta Phys. Sin. Ch. Ed. 2019.

18. Ye, L.; Qiu, C.; Lu, J.; Tang, K.; Jia, H.; Ke, M.; Peng, S.; Liu, Z. Making sound vortices by meta-surfaces. Amercian Inst. Phys. 2016.

19. Zhang, S.; Yin, L.; Fang, N. Focusing ultrasound with an acoustic metamaterial network. Phys. Rev. Lett. 2009, 102, 194301. [CrossRef] [PubMed]

20. Gao, H.; Gu, Z.; Liang, B.; Zou, X.; Yang, J.; Yang, J.; Cheng, J. Acoustic focusing by symmetrical self-bending beams with phase modulations. Appl. Phys. Lett. 2016, 108, 73501. [CrossRef]

21. Chen, L.; Hongxiang, S.; Shouqi, Y.; Jianping, X.; Jiao, Q. Acoustic focusing effect based on thermoacoustic phased array. Acta Phys. Sin. Ch. Ed. 2017, 66, 167-174.

22. Ge, Y.; Qian, J.; Sun, H. Broadband acoustic focusing effect based on heat source array. Tech. Acoust. $2016,3$.

23. Li, Y.; Assouar, B.M. Acoustic metasurface-based perfect absorber with deep subwavelength thickness. Appl. Phys. Lett. 2016, 108, 63502. [CrossRef]

24. Ma, G.; Yang, M.; Xiao, S.; Yang, Z.; Sheng, P. Acoustic metasurface with hybrid resonances. Nat. Mater. 2014, 13, 873-878. [CrossRef] [PubMed]

25. Jiang, X.; Li, Y.; Liang, B.; Cheng, J.; Zhang, L. Convert Acoustic Resonances to Orbital Angular Momentum. Phys. Rev. Lett. 2016, 117, 34301. [CrossRef]

26. Xie, Y.; Shen, C.; Wang, W.; Li, J.; Suo, D.; Popa, B.; Jing, Y.; Cummer, S.A. Acoustic Holographic Rendering with Two-dimensional Metamaterial-based Passive Phased Array. Sci. Rep. 2016, 6, 35437. [CrossRef]

27. Chen, Y.; Escalera Mendoza, A.S.; Griffith, D.T. Experimental and numerical study of high-order complex curvature mode shape and mode coupling on a three-bladed wind turbine assembly. Mech. Syst. Signal Pr. 2021, 160, 107873. [CrossRef]

28. Zeng, M.; Tan, B.; Ding, F.; Zhang, B.; Zhou, H.; Chen, Y. An experimental investigation of resonance sources and vibration transmission for a pure electric bus. Proc. Inst. Mech. Eng. Part D J. Automob. Eng. 2020, 234, 950-962. [CrossRef]

29. Yu, N.; Genevet, P.; Kats, M.A.; Aieta, F.; Tetienne, J.P.; Capasso, F.; Gaburro, Z. Light Propagation with Phase Discontinuities: Generalized Laws of Reflection and Refraction. Science 2011, 334, 333-337. [CrossRef] [PubMed] 
30. Zhou, X.; Hu, G. Dynamic effective models of two-dimensional acoustic metamaterials with cylindrical inclusions. Acta Mech. 2013, 224, 1233-1241. [CrossRef]

31. Wang, Z.; Chu, Y. Research progress of acoustic metasurface in China. EPJ Appl. Metamaterials 2019, 6, 5. [CrossRef]

32. Sugioka, K.; Xu, J.; Wu, D.; Hanada, Y.; Wang, Z.; Cheng, Y.; Midorikawa, K. Femtosecond laser 3D micromachining: A powerful tool for the fabrication of microfluidic, optofluidic, and electrofluidic devices based on glass. Lab. Chip. 2014, 14, 3447-3458 [CrossRef] [PubMed]

33. Cao, J.; Wu, Y.; Lu, D.; Fujimoto, M.; Nomura, M. Fundamental Machining Characteristics of Ultrasonic Assisted Internal Grinding of SiC Ceramics. Mater. Manuf. Process. 2014, 29, 557-563. [CrossRef]

34. Zhai, S.; Ding, C.; Chen, H.; Shen, F.; Luo, C.; Zhao, X. Anomalous Manipulation of Acoustic Wavefront with an Ultrathin Planar Metasurface. J. Vib. Acoust. 2016, 138, 041019. [CrossRef]

35. Takagi, R.; Koseki, Y.; Yoshizawa, S.; Umemura, S. Investigation of feasibility of noise suppression method for cavitation-enhanced high-intensity focused ultrasound treatment. Ultrasonics 2021, 114, 106394. [CrossRef]

36. Chahine, G.L.; Kapahi, A.; Choi, J.; Hsiao, C. Modeling of surface cleaning by cavitation bubble dynamics and collapse. Ultrason. Sonochem. 2016, 29, 528-549. [CrossRef] [PubMed] 